\title{
Complex Hydrological System Inflow Prediction using Artificial Neural Network
}

\author{
Petar MATIĆ*, Ozren BEGO, Matko MALEŠ
}

\begin{abstract}
Artificial neural networks have been successfully used to model and predict water flows for a few decades. Different network types have proven to work better in different cases and additional tools and algorithms have been implemented to improve those neural models. However, some problems still occur in certain cases. This paper deals with the limitation of complex hydrological system inflow prediction using artificial neural network and inflow time series. This limitation is called the prediction lag and it disables the model from giving accurate predictions. To eliminate the prediction lag and to extend prediction horizon an alternative input variable named forecasted precipitation frequency is proposed in addition to antecedent inflow time-series. Simulation results prove the efficiency of the proposed solution that enables time series neural network model for 7 th-day inflow prediction. This represents important information in operational planning of the hydrological system, used for short-term optimization of the system, e.g. optimization of the hydroelectric power plant operation.
\end{abstract}

Keywords: artificial neural network; complex hydrological system; forecasted precipitation frequency; inflow prediction; prediction lag

\section{INTRODUCTION}

Water and water streams represent a natural resource that is widely used in water supply, agriculture and transport, while water power is easily exploited for obtaining other energy forms, such as electrical energy in hydroelectric power plant (HPP). However, the wellknown fact of water cycle implies uncertainty of water availability in terms of quantity and time of occurrence. In order to optimize the use of water resources, hydrological models are used to make predictions of the hydrological variables, such as runoff, i.e. inflow, the most commonly predicted hydrological variable based on research published in [1].

The occurrence of runoff from the catchment is a complex function of different geographical, geological, biological, climatological and anthropological variables [2], where the relationship is usually non-linear [3]. Complexity and non-linearity of the hydrological system are further expressed in karst. Karst is a distinctive relief with a dominant underground flow through soluble rocks such as limestone, dolomite, gypsum (plaster), chalk and halite. The main problem in modelling runoff in karst is the fact that certain features of the catchment area, such as underground streams and reservoirs, are often unknown, or difficult to measure. Therefore, data based modelling is often a more convenient solution, regarding the appropriate measurements are available.

Artificial neural networks (ANNs) have the ability to model nonlinear systems based on the available data. The universal approximation capabilities of the multilayer perceptron (MLP) made it a popular choice for modelling various systems, where hydrological processes make no exception, as discussed in [1] and [4]. ANNs were found more than convenient, easy-to-use tool, which outperforms traditional statistical and physical models, as discussed in [5-8] and [9]. Some applications of ANNs to inflow modelling and prediction on karst landscape can be found in [9-12] and [13].

Although ANNs have been successfully used for shortterm inflow predictions, they do not represent a universal solution for modelling nonlinear systems and are highly data dependant. This can be supplemented by the research presented in [14] where different input variables were tested on the same catchment for various monthly predictions. In cases of weak connection between input and output data, the accuracy of predictions was compromised and similar occurred in this research.

In this paper, a short-term inflow prediction of the river Cetina to accumulation Peruća in Croatia using ANN and the available data was investigated. Based on available data obtained by Croatian Meteorological and Hydrological Service (CMHS), neural models were formed using different variables as inputs and the results were compared, as shown in section 4.1 of this paper. However, no matter which input variables were used, all of the analysed models suffered from a prediction lag deficiency which confirmed the existence of a prediction lag effect for the case study.

To eliminate prediction lag effect and extend prediction horizon an alternative input variable is introduced, as described in section 4.2. This variable is named forecasted precipitation frequency $\left(f_{k}\right)$, which has a meaning of a number of rain occurrences in period of $k$-days. The idea is to have a variable of a sufficient predictability (high probability) that "carries information from the future" and acts as a trigger, which, in conjunction with other input variables, enables the model to predict sudden changes of the output variable.

Furthermore, the prediction horizon represents an everlasting research topic, especially in case of inflow predictions for the purpose of hydrological system optimization, e.g. hydroelectric power plant (HPP) operation optimization. Prediction horizon is determined by the hydrological characteristics of the system, and it causes rapid decrease in prediction accuracy after optimal horizon is exceeded. A way to extend the prediction horizon is to use weather forecasts, i.e. precipitation forecasts, as in [15] and [16]. Unfortunately, precipitation forecasts are far from perfect and show high uncertainty [17]. The way of making weather forecasts more useful for prediction is to find a way to extract more reliable information from them, as proposed in this paper.

\section{THE PREDICTION LAG EFFECT}

The prediction lag effect can be described as model giving response with a delay, thus being unable to predict sudden changes of the output variable before they actually 
happen. This problem is associated with the prediction based on time series, where the occurrence of the prediction lag is explained by the lack of information that would indicate a sudden change of the predicted variable.

In simpler hydrological systems, this problem is usually solved just by adding antecedent rainfall to the set of input variables, as discussed in [4]. However, in more complex landscapes precipitation does not always reflect the runoff variations in the same manner. Precipitation information content may become inferior to the other input variables, e.g. antecedent inflow. In those cases, ANN easily discards precipitation during training, and uses only flow to approximate input-output mapping, as noted in [18]. In the same paper, it is pointed out that this phenomenon is still to be explored and adequate solution needs to be found.

Although it represents a serious drawback of the model, these timing errors (prediction lags) have received little attention, as in [7, 12, 19-23], which all present results with noticeable prediction lag but do not refer to the problem. These lagged predictions would of course reflect the overall performance of the model expressed in terms of numerical quality measure. Researchers would then turn to advanced algorithms in order to improve model performances, as in [23] and [24], failing to observe the actual problem, the lag effect.

\section{MODELLING METHOD: ARTIFICIAL NEURAL NETWORKS}

Inspired by biological neural networks, artificial neural networks (ANNs) represent a simplified mathematical model of the process that occurs in the brain of living beings, as explained in detail in [25]. Based on the type of neurons and the way they are connected, different ANNs have been developed over time and applied to different fields of science, ranging from pattern recognition to function approximation for modelling and prediction. The focus of this research was to enable inflow prediction for the complex hydrological system using an ANN as a modelling tool since it was not possible due to the prediction lag effect.

ANN was chosen as a modelling tool due to its advantages over similar data based modelling approaches. A static feed-forward ANN called Multi-Layer Perceptron (MLP) represents the most commonly used network for modelling processes. Although, it would be expected for dynamical ANNs, like recurrent neural networks (RNN) to be more suitable for modelling dynamical processes like inflow, some experiments show, e.g. [26], that MLP can equal or even outperform RNN. On the other hand, some of the researchers went the other way and produced fair results using RNN, as in [27]. Although there are papers comparing different type of networks, e.g. [28] and [29], there is no agreement on optimal ANN type and this topic remains open to further investigation. In this paper, an MLP neural network is used as the most common as well as convenient solution.

To define MLP model, it is necessary to determine a number of inputs $\left(N_{\mathrm{i}}\right)$, number of hidden neurons $\left(N_{\mathrm{h}}\right)$ and number of output neurons $\left(N_{\mathrm{o}}\right)$, i.e. number of output variables. Determination of the input variables is case dependent, and therefore it is discussed in more detail in section 4.3 of this paper. Number of output neurones is determined by the number of output variables, and in this case it is one neuron, to predict the inflow of the seventh day.

Optimal number of MLP layers was often the subject of research. However, it has been proven that two-layer structure is sufficient to approximate any practical function, given enough neurons in hidden layer [30]. Number of hidden neurons $\left(N_{\mathrm{h}}\right)$ determines the quality of the model, and therefore represents inevitable subject of research in model development process. To determine the optimal number of hidden neurons, in this paper the Bayesian regularization (BR) was used in addition to the experiment of training ANNs with different number of hidden neurons. BR, which can be found explained in [31], can be a useful tool in determining sufficient number of hidden neurons, making an improvement to the Levenberg-Marquardt (LM) algorithm, explained in [32] and [33]. LM was proven to be the fastest and most appropriate algorithm for training networks containing up to a few hundreds of adjustable parameters [34].

Therefore, MLP with two layers was used in this research to form a neural model for short-term inflow prediction. The network is trained with BR training algorithm in Matlab, using the data obtained from the CMHS

\section{CASE STUDY: PREDICTION OF THE RIVER CETINA INFLOW TO A RESORVOIR PERUĆA}

River Cetina basin, shown in Fig. 1, is the second largest basin of the Dinaric karst area with inflow into the Adriatic Sea and one of the most important water streams in Croatia. With a total length from source to estuary of $105 \mathrm{~km}$ and total drop in the amount of $385 \mathrm{~m}$, it represents a significant water and energy potential that is used for electric power production, water supply of the wide area, as well as in tourism and wastewater drainage.

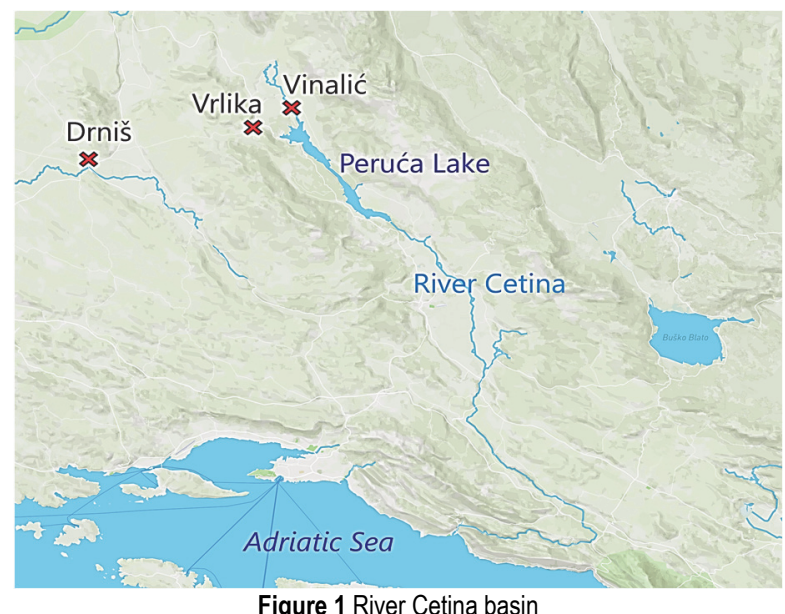

In the entire system, the most vulnerable to large inflows is reservoir Peruća, because of the accumulation capacity insufficient to accept large inflows during the period in which high water levels are present in the reservoir. By using inflow predictions up to seven days, negative effects of large water waves could be avoided. Furthermore, inflow predictions represent important information in operational planning of the hydrological system where inflow predictions are used for short-term optimization of the system such as operational planning of the HPP. 


\subsection{Data Analysis}

The available data include time series of average daily values of the variables measured at stations charted in Fig. 3 and noted in Tab. 1. The measurements were taken in 5year period from 2007 to 2012. All data were obtained from the CMHS, which represented the only available time series data at the beginning of the research.

Table 1 Available measured data

\begin{tabular}{|c|c|c|c|}
\hline Data No. & Variable & Location & Unit \\
\hline 1 & Flow & Vinalić & $\mathrm{m}^{3} \mathrm{~s}^{-1}$ \\
\hline 2 & Precipitation & Vrlika & $\mathrm{mm}$ \\
\hline 3 & Temperature & Drniš & ${ }^{\circ} \mathrm{C}$ \\
\hline 4 & Forecasted precipitation & Basin area & $\mathrm{mm}$ \\
\hline
\end{tabular}

To investigate available data influence on a modelled variable, i.e. inflow, and to alleviate input variable selection process, a correlation analysis was performed with results shown in Fig. 2.

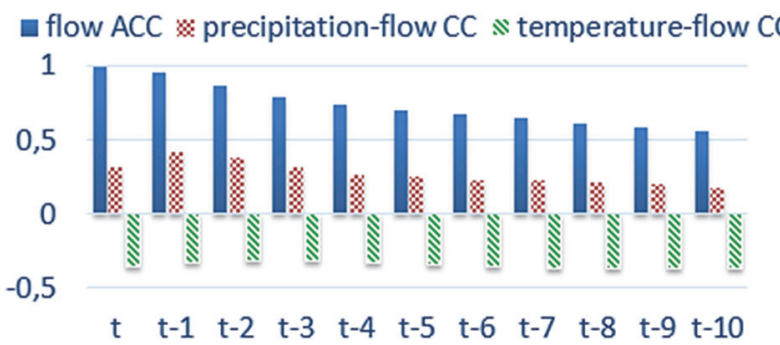

Figure 2 Correlation analysis of the precipitation, temperature and inflow time series

Based on the low value of the cross-correlation coefficient $(C C<0,42)$ it can be assumed that influence of the available precipitation data to the inflow is low. Correlation between available temperature measurements and flow has a negative value, which implies inversely proportional relationship, but still with low $C C$ values that imply low variable dependence. On the other hand, autocorrelation coefficients $(A C)$ between instant and antecedent inflow are of much higher values. Based on the presented analysis, it may be assumed that the only influential variable to the inflow prediction is the inflow itself. However, since the correlation analysis examines linear dependence between variables, the correlation-based assumption was tested on a model-based experiment, with graphical representation of the results shown in Fig. 3.

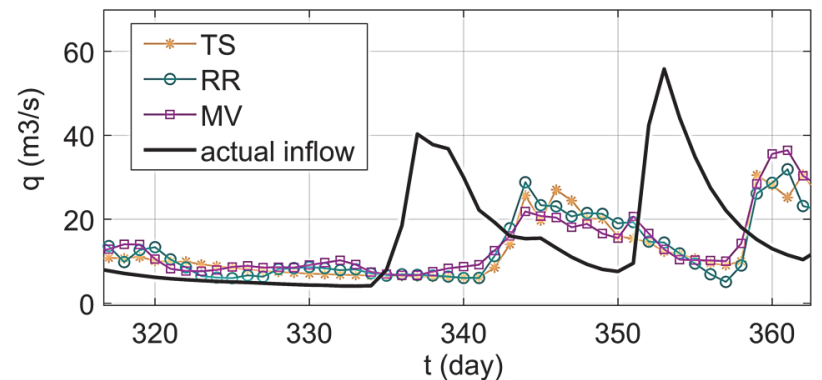

Figure 3 Comparison of TS, RR and MV model's prediction to an actual inflow of the seventh day

For the experiment purposes, three types of neural models ( $T S, R R$ and $M V$ ) were formed for one to nine days inflow prediction. A time series model (TS) that uses only antecedent inflows $\left(q_{t-1}, \ldots, q_{t-Q}\right)$ as inputs; a rainfallrunoff model $(R R)$ that, beside antecedent inflow, uses antecedent precipitation $\left(p_{t-1}, \ldots, p_{t-P}\right)$; and a multivariable model $(M V)$ that adds antecedent temperature $\left(t_{t-}\right.$ $\left.1, \ldots, t_{t-T}\right)$ to the set of inputs to predict future inflow $\left(q_{t}\right.$, $\left.\ldots, q_{t+k}\right)$.

Experiment results suggest that model makes no use of rainfall and temperature information, i.e. adding available rainfall and temperature data to the set of inputs could not prevent the prediction lag effect, as presented in Fig. 3. Therefore, to eliminate the prediction lag effect and preferably to extend the prediction horizon $(k)$ up to seven days an alternative input variable, a forecasted precipitation frequency, is introduced.

\subsection{Forecasted Precipitation Frequency $\left(f_{k}\right)$}

Forecasted precipitation frequency for the period of $k$ days $\left(f_{k}\right)$ is calculated based on weather forecasts obtained from CMHS. Forecasted precipitation quantities of a single day $(n)$ for the basin area $\left(F P_{Q}(n)\right)$ were acquired from simulation, using the ECMWF (European Centre for Medium Range Weather Forecasting) model.

Let $F P_{D}(n)$ be the binary function that says if there is a relevant rain quantity forecasted for day $n$ or not, as defined by Eq. (1). Then, $f_{k}$ is calculated as a sum of days with forecasted daily precipitation quantities higher than 1 $m m$ in the period of $k$ days, as defined by Eq. (2) and Eq. (3). Therefore, $f_{k}$ is a function that represents the number of days with forecasted significant rainfall occurrences in period of $k$ days and it is always a natural number.

$$
\begin{aligned}
& F P_{D}(n)=\left\{\begin{array}{ll}
0, & F P_{Q}(n)<1 \mathrm{~mm} \\
1, & F P_{Q}(n) \geq 1 \mathrm{~mm}
\end{array}\right\} \\
& f_{k}=\sum_{n=1}^{k} F P_{D}(n) \\
& f_{k}:\{0,1\} \rightarrow\{0,1, \ldots, k\} \subset \mathrm{N}
\end{aligned}
$$

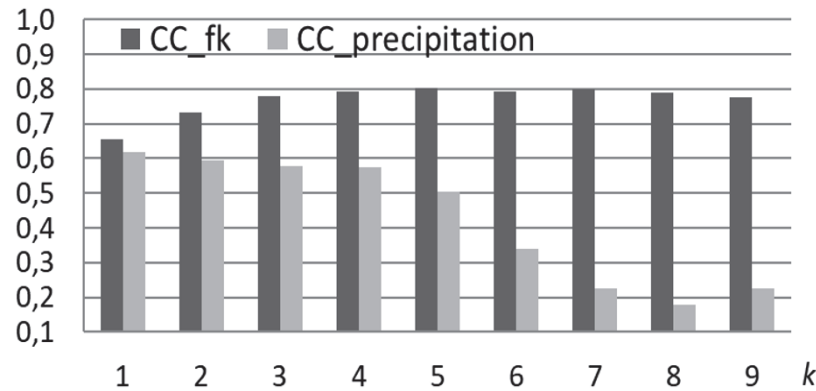

Figure 4 Correlation based reliability analysis of the forecasted precipitation and forecasted precipitation frequency $\left(f_{k}\right)$

It makes perfect sense to use precipitation forecast to predict future inflow. However, accuracy and reliability of precipitation forecast is relatively low, as noted in [8], and also confirmed by low value of correlation coefficients between forecasted precipitation and actual precipitation occurrences. As shown in Fig. 4 the correlation coefficient $(C C)$ for the first day forecast is low $(0,6)$, and it decreases with the increase of the prediction horizon $(k)$. 
Correlation results presented in Fig. 4 suggest that the forecasted precipitation quantity is useless information for the targeted prediction horizon of seven days (since $C C<$ 0,3 for $k>6$ ). On the other hand, correlation coefficients between forecasted precipitation frequency $\left(f_{k}\right)$ and actual precipitation frequency have higher values, as can be seen from the results of correlation analysis shown in Fig. 4. Correlation analysis shows high $C C$ values between the actual and forecasted precipitation frequency for the targeted prediction horizon $k \geq 7$, with the value of $C C=$ 0,8 for $k=7$, thus indicating high reliability of the created variable.

\subsection{Model Development}

MLP neural network with a structure of universal approximator, e.g. [25] and [30], is used to form a model for inflow prediction from $k=1$ to $k=9$ days ahead. The model is formed based on the direct method principle, shown in Fig. 5. Opposed to indirect method that uses $k$ networks to perform $k$-steps prediction, also investigated in this research, direct method uses a single network to predict $k$-step ahead, and it was used for the modelling simplicity since both methods were equally accurate. Antecedent inflow, $\left(q_{t-1}, \ldots, q_{t-Q}\right)$ and forecasted precipitation frequency $\left(f_{k}\right)$ are selected as inputs based on the model based research, meaning, the model uses a number of $Q+1$ inputs $\left(N_{\mathrm{i}}=Q+1\right)$ and one output $\left(N_{\mathrm{o}}=1\right)$.

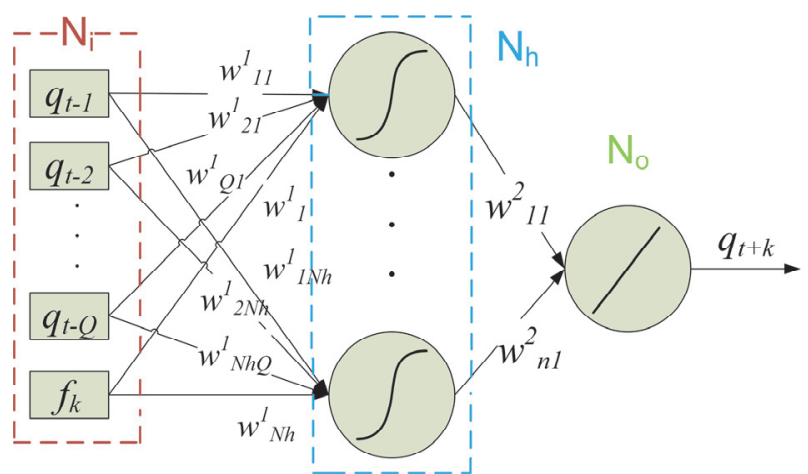

Figure 5 Direct method based model structure for multi-step prediction

To calibrate the model, i.e. to train the network, BR algorithm was used and the experiments were performed in order to determine the optimal number of input and hidden neurons, where sets of $Q \in[1,10]$ and $N_{\mathrm{h}} \in[1,20]$ were searched for optimal solution. Furthermore, since training outcome is affected by the initial values of the network parameters $(w)$, in order to bring the solution closer to a global minimum, a multi-start is applied. This means that each network structure with different initial values of the parameters $w$ is trained $I$ times, and the best-evaluated network is chosen to be a representative model. $I=10$ was used as the optimal number of consecutive network trainings based on a recommendation from [18].

The network was trained using 4-year daily data, which made 1460 examples for the training purposes and 1-year data, i.e. 365 examples for model evaluation, meaning that the prediction of the model was tested in unfamiliar cases. During the training, data has been divided randomly to "train", "test" and "validation" subsets in order to make use of early stopping method, as described in [34].
This method is used as prevention for overtraining, i.e. to improve generalization abilities of the model.

\subsection{Model Evaluation}

The paper [35] presents an overview of commonly used measures in hydrology and suggests the use of at least one relative and one absolute error measure for optimal model evaluation. Therefore, absolute measures: root mean squared error (RMSE), mean absolute error (MAE); and relative measures: Nash-Sutchllife coefficient of efficiency $(C E)$, percent bias $(P B I A S)$ and $R S R$ (RMSE to standard deviation ratio), are used in this paper to evaluate the quality of model prediction

Values of RMSE, MAE and RSR range from high values $(+\infty)$ to 0 , with lower values indicating lower errors. $C E$ values range from negative values $(-\infty)$ to 1 , where high positive values indicate a better fit. PBIAS measures the average propensity of the model to predict values smaller or larger than the measurements, as stated in [36]. The values of the PBIAS criteria range in the interval from 0 to $\pm 100 \%$, with smaller values indicating a better model performance. Positive values indicate a model bias toward underestimation, while negative values indicate a bias toward overestimation.

In paper [37], model classification is made based on the values of $C E, R S R$ and $P B I A S$ quality measures. The same classification is presented here in Tab. 2 and is used in this paper to determine acceptable model performance and optimal prediction horizon $(k)$.

Table 2 Model classification based on $C E, R S R$ and $P B I A S$ values

\begin{tabular}{|c|c|c|c|}
\hline Model quality & $C E$ & $R S R$ & $P B I A S$ \\
\hline Very good & $(0.75,1.00]$ & {$[0.00,0.50]$} & $< \pm 10 \%$ \\
\hline good & $(0.65,0.75]$ & $(0.50,0.60]$ & {$[ \pm 10, \pm 15) \%$} \\
\hline Satisfactory & $(0.5,0.65]$ & $(0.6,0.70]$ & {$[ \pm 15, \pm 25) \%$} \\
\hline Unsatisfactory & $\leq 0.5$ & $>0.7$ & $\geq \pm 25 \%$ \\
\hline
\end{tabular}

However, none of the numerical measures have shown the ability to penalize the prediction lag effect deficiency. Therefore, graphical evaluation imposes a mandatory supplement to the numerical evaluation of the model performance.

\section{SIMULATION RESULTS}

In order to determine model's quality and establish optimal prediction horizon, RMSE, MAE, CE, RSR and PBIAS are calculated for different prediction horizons, ranging from $k=1$ to $k=9$, and the results are presented in Tab. 3. For every $k$, an optimal number of input and hidden neurons was investigated to determine an optimal model structure as discussed in chapter 4.3.

Table 3 Evaluation of model prediction for horizons $k \in[1,9]$

\begin{tabular}{|c|c|c|c|c|c|c|}
\hline$k$ & $\begin{array}{c}\text { optimal } \\
\text { structure }\end{array}$ & $R M S E$ & $M A E$ & $C E$ & $R S R$ & $\begin{array}{c}\text { PBIAS / } \\
\%\end{array}$ \\
\hline 1 & $4-8-1$ & 1,267 & 0,564 & 0,922 & 0,278 & $-2,592$ \\
\hline 2 & $4-8-1$ & 2,730 & 1,139 & 0,690 & 0,558 & $-1,541$ \\
\hline 3 & $5-8-1$ & 3,270 & 1,677 & 0,605 & 0,698 & $-8,486$ \\
\hline 4 & $7-8-1$ & 3,648 & 1,957 & 0,555 & 0,645 & $-7,902$ \\
\hline 5 & $7-8-1$ & 3,649 & 2,183 & 0,578 & 0,611 & $-12,37$ \\
\hline 6 & $8-11-1$ & 3,676 & 2,272 & 0,582 & 0,635 & $-18,92$ \\
\hline 7 & $8-16-1$ & 3,879 & 2,488 & 0,542 & 0,650 & $-21,65$ \\
\hline 8 & $7-16-1$ & 4,523 & 2,808 & 0,346 & 0,659 & $-23,42$ \\
\hline 9 & $9-19-1$ & 4,761 & 3,054 & 0,321 & 0,758 & $-23,29$ \\
\hline
\end{tabular}


Based on the results from Tab. 3, it can be noted that prediction quality decreases as the prediction horizon rises. According to the criteria stated in Tab. 2, the model has satisfactory performances for the values up to $k \leq 7$. This implies that the model can be used for predictions of up to seventh day. Model's tendency toward overestimation can also be noted, since the value of PBIAS is negative.

In order to evaluate the model's ability to eliminate prediction lag deficiency, a graphical comparison of the model response and actual inflow of the seventh day is shown in Fig. 6. Data of the year 2012 were used to test the model, and the same data were not presented to the model during the calibration.

Based on the results displayed in Fig. 6 it can be noted that a neural model using the $f_{k}$ variable can predict inflow variations seven days ahead without lag effect in most cases. Although model response varies in terms of accuracy, most of the moments of significant changes of the inflow are accurately predicted, which proves the efficiency of the proposed solution.

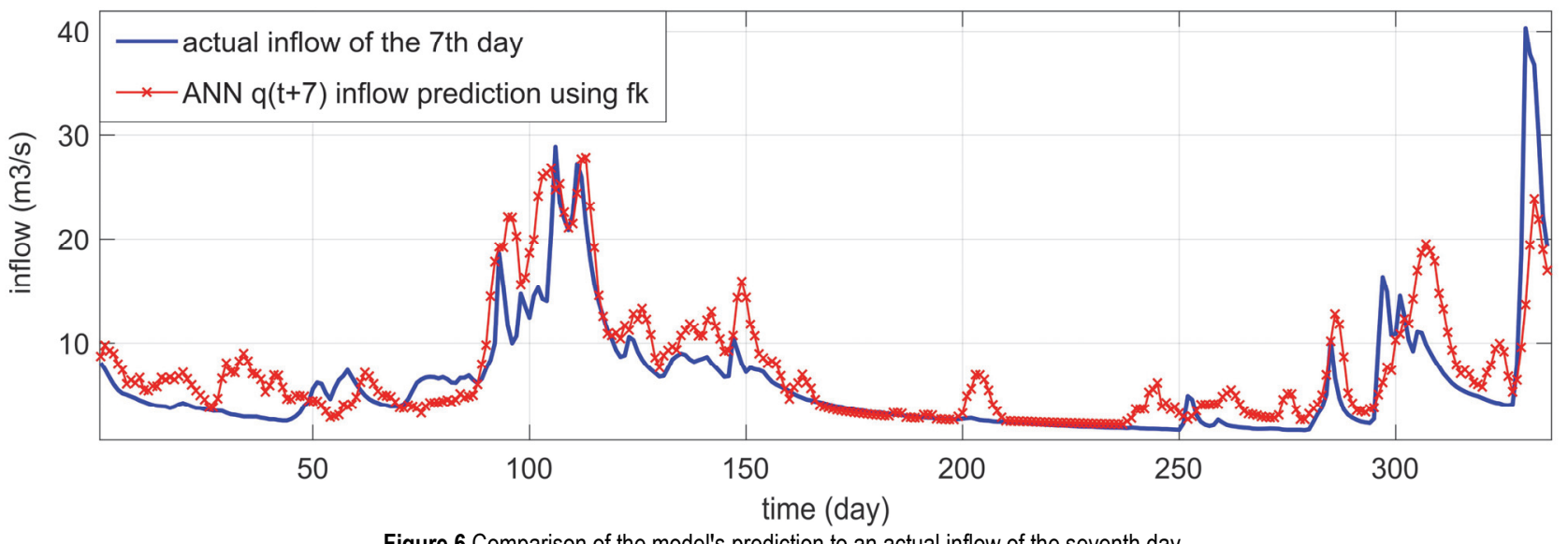

Figure 6 Comparison of the model's prediction to an actual inflow of the seventh day

\section{CONCLUSION}

Based on the experiments performed, it can be concluded that ANNs have justified the reputation of a flexible tool, capable of modelling complex, nonlinear systems, but also pointed out that ANNs greatly depend on the data that can be collected from the modelled system.

For the case of the river Cetina a prediction lag effect was detected, making the model unable to predict sudden changes of the inflow, as described in chapter 4.1. Furthermore, none of the available measured variables, i.e. precipitation and temperature, could eliminate this lag effect when added to the set of inputs, nor could it contribute to the model's performance quality. Therefore, in order to eliminate the prediction lag effect and to extend prediction horizon of the model, forecasted precipitation frequency for the prediction horizon of $k$ days $\left(f_{k}\right)$ was added to the antecedent inflow time series at the input of the model.

Based on the results presented in section 5 of this paper, it can be concluded that the variable $f_{k}$ can help to predict sudden changes of the inflow, thus solve the problem of the lag effect for the case study of the river Cetina. Experimental results indicate that sufficiently accurate inflow prediction for the seventh day is possible using the proposed solution, which makes this model suitable for short term optimization of the hydrological system.

It can be emphasised that although the prediction lag effect is eliminated and numerical measures fairly evaluate the model, it is assumed that further improvements of the prediction accuracy could be made. Therefore, the focus of further investigation should be on the improvement of the prediction accuracy and the application of this solution to the optimization of a hydro power plant operation.

\section{Acknowledgements}

The author of this paper would like to express gratitude to the employers of the Croatian Meteorological and Hydrological Service for providing the data that enabled this work.

\section{REFERENCES}

[1] Maier, H. R. et al. (2010). Methods used for the development of neural networks for the prediction of water resource variables in river systems: Current status and future directions. Environmental Modelling \& Software, 25, 891909. https://doi.org/10.1016/j.envsoft.2010.02.003

[2] Zugaj, R. (2010). High Waters of Small Basins. University of Zagreb, Faculty of Mining, Geology and Petroleum Engineering, Zagreb.

[3] Sivakumar, B. (2009). Nonlinear dynamics and chaos in hydrologic systems: latest developments and a look forward. Stochastic Environmental Research and Risk Assessment, 23(7), 1027-1036. https://doi.org/10.1007/s00477-008-0265-z

[4] Abrahart, R. J., Kneale, P. E., \& See, L. M. (2004). Neural Networks for Hydrological Modelling. CRC Press, London, UK. https://doi.org/10.1201/9780203024119

[5] Agarwal, A. \& Singh, R.D. (2004). Runoff Modelling Through Back Propagation Artificial Neural Network with Variable Rainfall-Runoff Data. Water Resources Management, 18, 285-300. https://doi.org/10.1023/B:WARM.0000043134.76163.b9

[6] Bhadra, A. et al. (2010). Rainfall-Runoff Modelling: Comparison of Two Approaches with Different Data Requirements. Water Resources Management, 24, 37-62. https://doi.org/10.1007/s11269-009-9436-z

[7] Rasouli, K., Hsieh, W. W., \& Cannon, A. J. (2012). Daily streamflow forecasting by machine learning methods with weather and climate inputs. Journal of Hydrology, 414, 284293. https://doi.org/10.1016/j.jhydrol.2011.10.039 
[8] Taormina, R., Chau, K., \& Sethi, R. (2012). Artificial Neural Network simulation of hourly groundwater levels in a coastal aquifer system of the Venice lagoon. Engineering Applications of Artificial Intelligence, 25(8), 1670-1676. https://doi.org/10.1016/j.engappai.2012.02.009

[9] Trichakis, I. C., Nikolos, I. K., \& Karatzas, G. P. (2009). Optimal selection of artificial neural network parameters for the prediction of a karstic aquifer's response. Hydrological Processes, 23, 2956-2969. https://doi.org/10.1002/hyp.7410

[10] Lallahem, S. \& Mania, J. (2003). A Nonlinear RainfallRunoff Model using Neural Network Technique: Example in Fractured Porous Media, Mathematical and Computer Modelling, 37, 1047-1061. https://doi.org/10.1016/s0895-7177(03)00117-1

[11] $\mathrm{Hu}, \mathrm{C}$. et al. (2008). Simulation of spring flows from a karst aquifer with an artificial neural network. Hydrological Processes, 22, 596-604. https://doi.org/10.1002/hyp.6625

[12] Kong a Siou, L., et al. (2012). Optimization of the generalization capability for rainfall-runoff modelling by neural networks: The case of the Lez aquifer (southern France). Environmental Earth Sciences 65(8), 2365-2375. https://doi.org/10.1007/s12665-011-1450-9

[13] Paleologos, E. K. et al. (2013). Neural network simulation of spring flow in karst environments. Stochastic Environmental Research and Risk Assessment, 27(8), 1829-1837. https://doi.org/10.1007/s00477-013-0717-y

[14] Mishra, P. K. \& Karmakar, S. (2019). Performance of optimum neural network in rainfall-runoff modeling over a river basin. International Journal of Environmental Science and Technology, 16(3), 1289-1302. https://doi.org/10.1007/s13762-018-1726-7

[15] Paravana, D. et al. (2004). Improvements to the water management of a run-of-river HPP reservoir: methodology and case study. Control Engineering Practice, 12(4), 377385. https://doi.org/10.1016/S0967-0661(03)00106-0

[16] Zhang, J. et al. (2009). Daily reservoir inflow forecasting combining QPF into ANNs model. Hydrol. Earth Syst. Sci. Discuss., 6, 121-150. https://doi.org/10.5194/hessd-6-121-2009

[17] Fan, F. M. et al. (2015). Verification of inflow into hydropower reservoirs using ensemble forecasts of the TIGGE database for large scale basins in Brazil. Journal of Hydrology: Regional Studies, 4, 196-227. https://doi.org/10.1016/j.ejrh.2015.05.012

[18] de Vos, N. J. \& Rientjes, T. H. M. (2005). Constraints of artificial neural networks for rainfall-runoff modelling: trade-offs in hydrological state representation and model evaluation. HESS9, 111-126. https://doi.org/10.5194/hess-9-111-2005

[19] Riad, S., Mania, J., Bouchaou, L., \& Najjar, Y. (2004). Rainfall-Runoff Model Using an Artificial Neural Network Approach. Mathematical and Computer Modelling, 40, 839846. https://doi.org/10.1016/j.mcm.2004.10.012

[20] Wang, W. et al. (2006). Forecasting daily streamflow using hybrid ANN models. Journal of Hydrology, 324, 383-399. https://doi.org/10.1016/j.jhydrol.2005.09.032

[21] Kişi, Ö. (2007). Streamflow Forecasting Using Different Artificial Neural Network Algorithms. Journal of Hydrologic Engineering, 12(5), 532-539. https://doi.org/10.1061/(ASCE)1084-0699(2007)12:5(532)

[22] Sattari, M. T., Yurekli, K., \& Pal, M. (2012). Performance evaluation of artificial neural network approaches in forecasting reservoir inflow. Applied Mathematical Modelling, 36, 2649-2657. https://doi.org/10.1016/j.apm.2011.09.048

[23] Meshram, S. G., Ghorbani, M. A., Shamshirband, S. et al. (2019). River flow prediction using hybrid PSOGSA algorithm based on feed-forward neural network. Soft Computing, 23(20), 10429-10438. https://doi.org/10.1007/s00500-018-3598-7
[24] Shafaei, M. \& Kisi, O. (2017). Predicting river daily flow using wavelet-artificial neural networks based on regression analyses in comparison with artificial neural networks and support vector machine models. Neural Computing and Applications, 28(1), 15-28. https://doi.org/10.1007/s00521-016-2293-9

[25] Haykin, S. (1999). Neural Networks: A Comprehensive Foundation. Pearson Education Inc. Second Edition, 2004.

[26] Tran, H. D., Muttil, N., \& Perera, B. J. C. (2011). Investigation of artificial neural network models for streamflow forecasting. $19^{\text {th }}$ International Congress on Modelling and Simulation, 12-16.

[27] Kratzert, F. et al. (2018). Rainfall-runoff modelling using long short-term memory (LSTM) networks. Hydrol. Earth Syst. Sci. Discuss, 22(11), 6005-6022. https://doi.org/10.5194/hess-22-6005-2018

[28] Liu, F., Xu, F., \& Yang, S. (2017). A flood forecasting model based on deep learning algorithm via integrating stacked autoencoders with BP neural network. IEEE $3^{\text {rd }}$ International conference on multimedia big data (BigMM), 58-61. https://doi.org/10.1109/BigMM.2017.29

[29] Alizadeh, Z. et al. (2018). Assessment of machine learning techniques for monthly flow prediction. Water, 10(11), 1676. https://doi.org/10.3390/w10111676

[30] Cybenko, G. (1989). Approximation by Superpositions of a Sigmoidal Function. Mathematics of Control Signals Systems, 2, 303-314. https://doi.org/10.1007/BF02551274

[31] Foresee, F. D. \& Hagan, M. T. (1997). Gauss-Newton approximation to Bayesian learning. ICNN'97, 3, 1930-1935. https://doi.org/10.1109/ICNN.1997.614194

[32] Hagan, M. T. \& Menhaj, M. B. (1994). Training Feedforward Networks with the Marquardt Algorithm. IEEE Transactions on Neural Networks, 5(6), 989-993. https://doi.org/10.1109/72.329697

[33] Yu, H. \& Wilamowski, B. M. (2011). Levenberg-Marquardt Training. Industrial Electronics Handbook, 5, 12-15. https://doi.org/10.1201/b10604-15

[34] Beale, M. H., Hagan, M. T., \& Demuth, H. B. (2010). Neural Network Toolbox TM User's Guide. The MathWorks, Inc.

[35] Legates, D. R. \& McCabe Jr., G. J. (1999). Evaluating the use of "goodness-of-fit" measures in hydrologic and hydroclimatic model validation. Water Resources Research, 35(1), 233-241. https://doi.org/10.1029/1998WR900018

[36] Gupta, H. V. et al. (1999). Status of Automatic Calibration for Hydrologic Models: Comparison with Multilevel Expert Calibration. Journal of Hydrologic Engineering, 4(2), 135145. https://doi.org/10.1061/(ASCE)1084-0699(1999)4:2(135)

[37] Moriasi, D. N. et al. (2007). Model Evaluation Guidelines for Systematic Quantification of Accuracy in Watershed Simulations. Transactions of the ASABE, 50(3), 885-900. https://doi.org/10.13031/2013.23153

\section{Contact information}

Petar MATIĆ, PhD, Assistant Professor

University of Split, Faculty of Maritime Studies,

R. Boskovica 37, 21000 Split, Croatia

E-mail: pmatic@pfst.hr

Ozren BEGO, PhD, Associate Professor

University of Split, Faculty of Electrical Engineering,

Mechanical Engineering and Naval Architecture (FESB)

R. Boskovica 32, 21000 Split, Croatia

E-mail: obego@fesb.hr

\section{Matko MALES}

University of Split, Faculty of Maritime Studies,

R. Boskovica 37, 21000 Split, Croatia

E-mail: mmales@pfst.hr 